\title{
Parler d'alcool en toute décontraction, sans frustration
}

\author{
Regula Hälga, Stefan Neuner-Jehle ${ }^{b}$ \\ a Infodrog; ${ }^{b}$ Collège de Médecine de Premier Recours
}

La consommation excessive d'alcool engendre de grandes souffrances et coûte cher. Plus les problèmes d'alcool sont repérés tôt, plus il est facile de modifier un comportement addictif. En abordant la consommation d'alcool avec leurs patients, les médecins jouent un rôle crucial dans le dépistage. Toutefois, cette démarche ne s'improvise pas et ne va pas de soi.

L'abus d'alcool rend malade: aucun organe, ou presque, n'est épargné. Le foie et l'appareil digestif sont les plus fortement touchés. Un décès sur douze en Suisse est lié à la consommation d'alcool, ce qui représentait par exemple environ 1600 personnes, de 15 à 74 ans, en 2011. Et dans trois cas sur cinq, une consommation chronique élevée en est la cause [1]. L'abus d'alcool engendre, en outre, des coûts annuels de l'ordre de 4,2 milliards de francs [2].

Parallèlement, $75 \%$ de la population en Suisse déclare accepter les incitations à adopter un comportement sain [3]. Les patients apprécient également que leur médecin aborde la consommation d'alcool, en particulier lorsque cela a à voir avec leur bien-être ou leur santé [4]. Mais comment éviter que la discussion ne génère des frustrations? Il suffit que les médecins soient motivés, qu'ils s'intéressent tout simplement à la consommation d'alcool de leurs patients et qu'ils réfléchissent, avec eux, à son impact sur le quotidien et sur la santé de ces derniers.

\section{Dépistage}

La consommation d'alcool est un plaisir pour la plupart des gens. Et il n'y a rien de mal à ça. Mais à partir de combien estime-t-on que c'est trop? La Commission fédérale pour les problèmes liés à l'alcool (CFAL) a émis des repères concernant la consommation d'alcool et considère qu'elle présente peu de risques dans la configuration suivante: Les hommes adultes en bonne santé peuvent boire deux à maximum trois verres standard d'alcool par jour, et les femmes, quant à elles, peuvent en consommer un à maximum deux par jour. En outre, de manière générale, il est conseillé de prévoir des jours sans alcool chaque semaine. Lors d'occasions spéciales plus arrosées, les hommes ne devraient pas boire plus de cinq verres, et les femmes, pas plus de quatre. Plus une consommation d'alcool à risque est repérée tôt, plus il y a de chances d'adapter son comportement ou de réduire sa consommation.

\begin{tabular}{|c|c|c|c|c|c|}
\hline Points & 0 & 1 & 2 & 3 & 4 \\
\hline $\begin{array}{l}\text { Avec quelle fréquence consommez-vous } \\
\text { des boissons alcooliques? }\end{array}$ & jamais & $\begin{array}{l}\text { une fois par mois / } \\
\text { ou moins }\end{array}$ & $\begin{array}{l}2 \text { à } 4 \text { fois } \\
\text { par mois }\end{array}$ & $\begin{array}{l}2 \text { à } 4 \text { fois } \\
\text { par semaine }\end{array}$ & $\begin{array}{l}4 \text { fois par semaine } \\
\text { ou plus }\end{array}$ \\
\hline $\begin{array}{l}\text { Lorsque vous consommez des boissons } \\
\text { alcooliques, combien en consommez-vous } \\
\text { un jour typique où vous buvez? }\end{array}$ & $1-2$ & $3-4$ & $5-6$ & $7-9$ & 10 ou plus \\
\hline
\end{tabular}

Total points (0-12):

Remarque: une boisson correspond à $10 \mathrm{~g}$ d'alcool.

Evaluation:

A partir de cinq points pour les hommes, quatre pour les femmes, on peut parler de risque élevé ou de consommation à risque. 


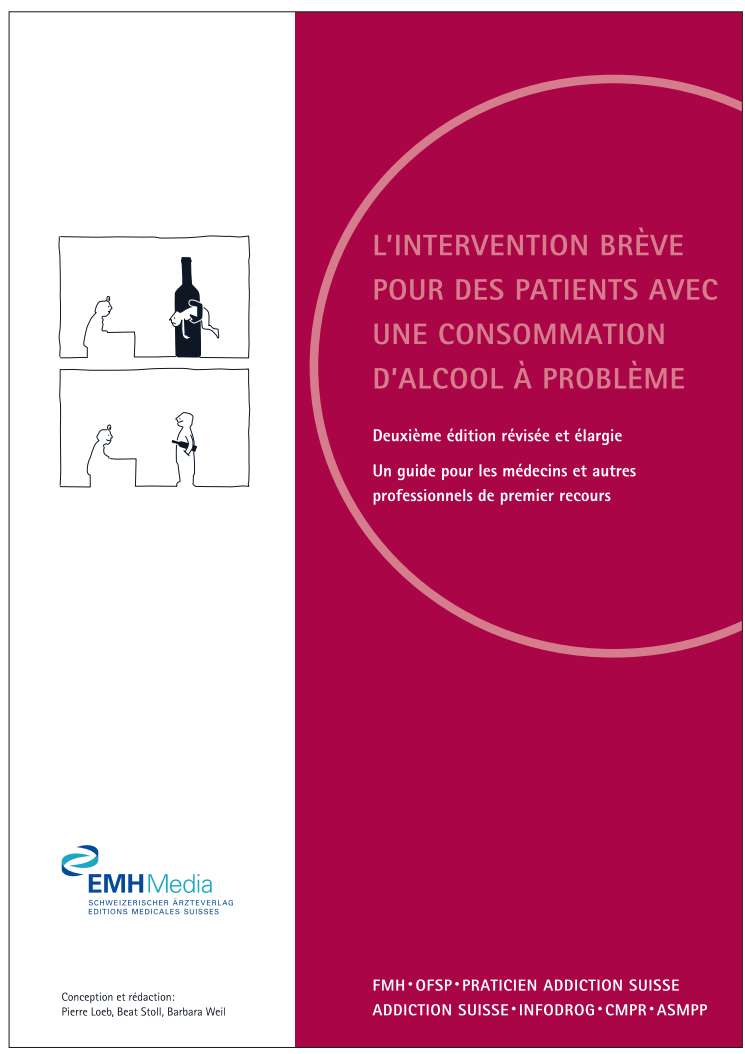

Figure 1: La brochure L'intervention brève pour des patients avec une consommation d'alcool à problème [7] peut être téléchargée sur www.praticien-addiction.ch ou commandée, par courriel, auprès du secrétariat de l'Académie suisse pour la médecine psychosomatique et psychosociale (ASMPP) contre une participation de 5 francs (frais de port et d'emballage en sus).

\section{Aborder la consommation d'alcool...}

Mais comment parler de comportement sain avec un patient ou même de sa consommation d'alcool? Le médecin peut envisager d'aborder ce sujet avec un patient à l'occasion d'un contrôle de routine, dans le cadre d'une discussion sur les habitudes de vie ou en cas de troubles pouvant (entre autres) être liés à une consommation d'alcool à risque.

L'attitude du praticien est déterminante pour le déroulement de l'entretien. Le patient est expert de son mode de vie et, en principe, concerné par son bien-être. Le médecin doit donc s'adresser à lui d'égal à égal: «Puis-je vous poser quelques questions sur votre consommation d'alcool?» Si le patient refuse, le médecin doit l'accepter. Il est important que ce dernier se montre disposé en tout temps à aborder ce sujet. En règle générale, les patients apprécient que leur médecin parle avec eux de leur consommation d'alcool.

Il est possible de repérer, de manière simple et fiable, si un patient présente une consommation à risque en lui posant les trois questions de l'AUDIT-C (tab. 1) [5]. Le médecin peut également lui remettre le questionnaire AUDIT en lui proposant de le remplir et d'en discuter lors de la prochaine consultation. Ou il peut lui suggérer de faire des tests en ligne; les résultats transmis par voie électronique sont généralement perçus comme étant moins «invasifs».

\section{... et intervenir}

Lorsque le médecin identifie une consommation à risque, il doit informer objectivement son patient sur les risques et les conséquences possibles; il doit éviter tout discours moralisateur. Il est important de donner la possibilité au patient de remettre en question ses propres habitudes de consommation, de le laisser poser ses questions et d'y apporter des réponses. L'intervention brève se prête particulièrement bien dans le cadre du cabinet médical. Elle a été objet de nombreuses recherches en médecine de premier recours qui ont démontré de bons résultats en termes de réduction de la consommation [6]. En pratique, l'intervention brève consiste en une série limitée d'entretiens courts. Elle s'inspire de l'entretien motivationnel et peut être structurée de manière très variée. L'intervention brève nécessite peu de temps, ce qui représente un grand avantage vu le manque de temps pour la plupart des médecins. Des connaissances et certaines aptitudes sont essentielles pour garantir son succès.

La brochure L'intervention brève pour des patients avec une consommation d'alcool à problème [7] (fig. 1) fournit aux médecins non seulement des informations de base sur l'alcool, mais également des astuces concrètes pour aborder le sujet et mener l'intervention brève.

\section{Mener une intervention brève motivationnelle}

Pour mettre cette méthode en pratique, les médecins peuvent également suivre des formations continues. Un groupe de travail interdisciplinaire composé du

\section{Informations et campagne}

II existe de nombreuses possibilités, tant pour les médecins que pour les patients, de s'informer sur les problèmes liés à l'alcool, par exemple sur www.praticien-addiction.ch (plus particulièrement pour les médecins et les spécialistes), sur www.addiction suisse.ch ou sur www.safezone.ch.

La campagne de prévention contre les problèmes liés à I'alcool menée par l'Office fédéral de la santé publique et ses partenaires informe de manière ludique sur les risques et les effets de I'alcool par un quiz disponible en ligne ou sur papier et incite à se poser la question: «Trop, c'est combien?» Les cabinets médicaux, les hôpitaux et toute autre organisation intéressée peuvent commander gratuitement le quiz. www.alcohol-facts.ch 
Forum de médecine de l'addiction de Suisse orientale (FOSUMOS), du Collège de médecine de premier recours (Programme Coaching Santé), de la Zürcher Fachstelle zur Prävention des Alkohol- und Medikamentenmissbrauchs (ZüFAM) et de la Zürcher Fachstelle für Alkoholprobleme (ZFA) propose aux médecins des cours de sensibilisation et des formations continues sur l'intervention brève consacrée à l'alcool. L'offre comprend des présentations ( 1 à $2 \mathrm{~h}$ ), des cours d'une demi-journée et des séminaires d'une journée. L'objectif est de dispenser aux médecins des connaissances et des compétences leur permettant d'inciter leurs patients à changer de mode de vie et de les soutenir de manière efficace dans leur démarche. Les médecins peuvent non seulement exploiter ces compétences pour aborder la consommation problématique d'alcool mais également une multitude d'autres comportements influant sur la santé [8].

\section{Autres projets pour la promotion de l'intervention brève}

Infodrog, la Centrale nationale de coordination des addictions, et le Programme national alcool contribuent au financement non seulement des activités proposées par le groupe de travail interdisciplinaire, mais aussi du projet pilote «Dépistage de la consommation d'alcool à risque et intervention brève électronique au cabinet médical» de l'Hôpital universitaire de Lausanne auquel participent huit cabinets médicaux; les patients présents dans la salle d'attente sont invités à répondre à des questions sur leur consommation d'alcool à l'aide d'une tablette et à discuter du résultat avec le médecin pendant la consultation. Les premiers résultats sont attendus pour l'automne 2016.

\section{Conclusion}

Un travail de sensibilisation précoce et une communication explicite sur la consommation problématique d'alcool constituent une tâche importante pour les médecins généralistes. Que cette tâche paraisse difficile ou soit vécue avec enthousiasme dépend principalement de la capacité à pouvoir parler ouvertement au patient et des compétences dont on dispose en communication ou encore de la motivation à les acquérir.
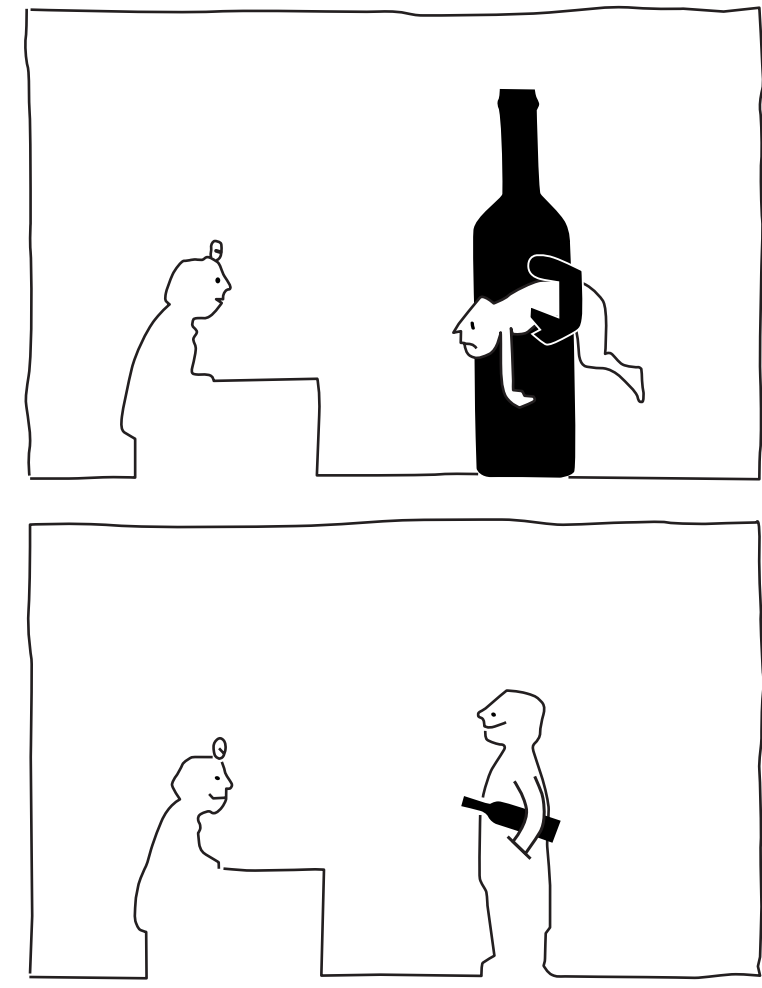

Références

1 Marmet S, Gmel G, Gmel G, Frick H, Rehm J, Shield KDC. (2013). Alcohol-attributable mortality in Switzerland between 1997 and 2011. Lausanne: Addiction Suisse.

2 Fischer B, Telser H, Widmer P, Leukert K. (2014). Coûts liés à l'alcool en Suisse. Etude menée sur mandat de l'Office fédéral de la santé publique. Olten: Polynomics.

3 pharma:ch 1/2008 Prévention: un investissement dans la santé et la prospérité du pays, voir sur www.interpharma.ch/fr/systemede-sante/2471-la-promotion-de-la-sante-revet-de-plus-enplus-dimportance, accès le 28.7.2016.

4 Daeppen JB, Gaume MJ. (2006). Implémentation et dissémination de l'intervention brève pour la consommation d'alcool à risque en médecine de premier recours: Evaluation du projet partiel «médecins» du Programme national alcool «Ça débouche sur quoi?» Rapport final rédigé sur mandat de l'Office fédéral de la santé publique. Lausanne: CHUV.

5 Bush K, Kivlahan DR, McDonell MB. (1998). The AUDIT alcohol consumption questions (AUDIT-C): an effective brief screening test for problem drinking. Ambulatory Care Quality Improvement Project (ACQUIP). Alcohol Use Disorders Identification Test. Arch Intern Med;158:1789-95.

6 Bertholet N, Daeppen JB, Wietlisbach V, Fleming M, Burnand B. (2005). Reduction of Alcohol Consumption by Brief Alcohol Intervention in Primary Care - Systematic Review and Meta-analysis. Arch Intern Med. 2005;165:986-95.

7 Editeur: FMH, OFSP, Médecin-Praticien, Addiction Suisse, Infodrog, CMPR et ASMPP, 2014.

8 Offres de formation sous www.zfa.ch/index.php/fortbildungaerzte.html 